\title{
Lignin resists high-intensity electron beam irradiation.
}

Oliver Sarosi ${ }^{\mathrm{a}}$, Irina Sulaeva ${ }^{\mathrm{a}}$, Elisabeth Fitz ${ }^{\mathrm{a}}$, Ivan Sumerskii ${ }^{\mathrm{b}}$, Markus Bacher ${ }^{\mathrm{b}}$, Antje Potthast ${ }^{\mathrm{b} *}$

a Kompetenzzentrum Holz GmbH, Altenbergerstraße 69, A-4040 Linz

b Institute of Chemistry of Renewable Resources, Department of Chemistry, University of Natural Resources and Life Sciences, Konrad-Lorenz-Straße 24, A-3430 Tulln

*Phone: +43 1 47654-77412, 77471, e-Mail: antje.potthast@boku.ac.at

Keywords: milled wood lignin, kraft, lignosulfonate, radical, antioxidant, molar mass

\section{Table of Contents}

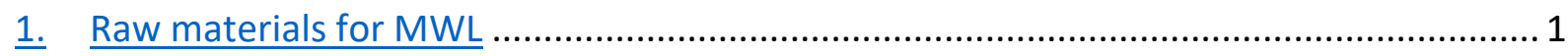

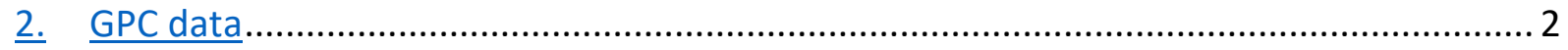

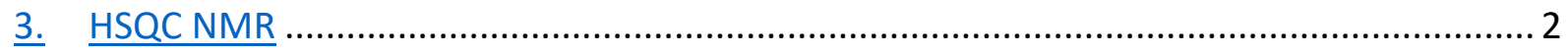

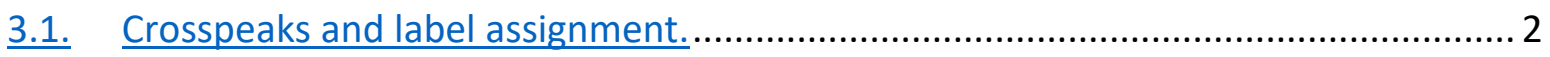

3.2. Volume integrals of characteristic lignin bonds................................................. 3

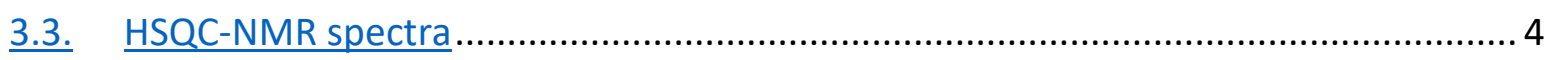

\section{Raw materials for MWL}

Table 1: Chemical composition of wood used for milled wood lignin production.

\begin{tabular}{|l|l|l|l|}
\hline Wood & $\begin{array}{l}\text { Fagus sylvatica } \\
\text { short-term storage } \\
\text { (A) }\end{array}$ & $\begin{array}{l}\text { Fagus sylvatica long- } \\
\text { term storage (B) }\end{array}$ & $\begin{array}{l}\text { Eucalyptus grandis } x \\
\text { urophylla (C) }\end{array}$ \\
\hline Dry content & $55.2 \%$ & $57.3 \%$ & $51.6 \%$ \\
\hline Acetone extractives & $0.50 \%$ & $0.98 \%$ & $1.04 \%$ \\
\hline Ash content & $0.08 \%$ & $0.33 \%$ & $0.33 \%$ \\
\hline Klason-lignin & $21.9 \%$ & $21.1 \%$ & $20.2 \%$ \\
\hline Acid soluble lignin & $3.0 \%$ & $3.5 \%$ & $3.0 \%$ \\
\hline Glucan & $49.1 \%$ & $48.5 \%$ & $40.7 \%$ \\
\hline Other carbohydrates & $14.8 \%$ & $22.6 \%$ & $20.3 \%$ \\
\hline
\end{tabular}




\section{GPC data}

Table 2: Calculated statistical moments of lignin samples before and after irradiation from gel permeation chromatography.

\begin{tabular}{|l|l|l|l|l|}
\hline Sample & $\mathrm{M}_{\mathrm{n}}(\mathrm{kDa})$ & $\mathrm{M}_{\mathrm{w}}(\mathrm{kDa})$ & $\mathrm{M}_{\mathrm{z}}(\mathrm{kDa})$ & $\bigoplus$ \\
\hline $\begin{array}{l}\text { MWL A } \\
\text { reference }\end{array}$ & 2.1 & 14.6 & 77.3 & 6.9 \\
\hline MWL A 200 & 2.0 & 17.7 & 139.0 & 8.8 \\
\hline MWL B reference & 26.8 & 608.6 & 5689.4 & 22.8 \\
\hline MWL B 200 & 28.8 & 538.2 & 5256.3 & 18.7 \\
\hline MWL C reference & 3.0 & 16.5 & 67.7 & 5.5 \\
\hline MWL C 200 & 3.3 & 16.3 & 79.5 & 4.9 \\
\hline KL reference & 0.5 & 1.4 & 5.5 & 3.1 \\
\hline KL 200 & 0.4 & 1.5 & 4.7 & 3.4 \\
\hline LS reference & 1.1 & 8.0 & 41.6 & 7.2 \\
\hline LS 200 & 1.0 & 7.9 & 48.0 & 8.1 \\
\hline
\end{tabular}

\section{HSQC NMR}

\subsection{Crosspeaks and label assignment.}

Table 3: Label, chemical shift and assignments of crosspeak signals in HSQC NMRs of all lignin samples.

\begin{tabular}{|c|c|c|c|}
\hline Crosspeak Label & H (ppm) & $C$ (ppm) & Assignment \\
\hline$A-\beta$ & 3.05 & 53.5 & $C_{\beta}-H_{\beta}$ in $\beta-\beta^{\prime}$ (resinol) substructures (A) \\
\hline $\mathrm{MeO}$ & 3.72 & 55.7 & Methoxy groups \\
\hline B- $-\gamma$ & 4.09 & 61.7 & $\mathrm{C}_{Y}-\mathrm{H}_{\gamma}$ in $p$-hydroxycinnamyl alcohol end groups (B) \\
\hline$C-\gamma$ & 3.69 & 59.5 & $\mathrm{C}_{Y}-\mathrm{H}_{\gamma}$ in $\beta-\mathrm{O}-4^{\prime}$ substructures $€$ and others \\
\hline$D-\beta$ & 2.75 & 60.1 & $\mathrm{C}_{\beta}-\mathrm{H}_{\beta}$ in $\beta-1^{\prime}$ (spriodienone) substructures (D) \\
\hline $\mathrm{X}-5$ & 3.31 & 63.8 & $\mathrm{C}_{5}-\mathrm{H}_{5}$ in Xylopyranose \\
\hline$\alpha-S A(\alpha)$ & 3.98 & 67.7 & $\mathrm{C}_{\alpha}-\mathrm{H}_{\alpha}$ with a sulfonic acid group in $\alpha$ position \\
\hline$A-\gamma 1$ & 4.17 & 71.1 & $\mathrm{C}_{\gamma 1}-\mathrm{H}_{\gamma 1}$ in $\beta-\beta^{\prime}$ (resinol) substructures (0) \\
\hline$A-\gamma^{2}$ & 3.79 & 71.0 & $\mathrm{C}_{\gamma 2}-\mathrm{H}_{\gamma 2}$ in $\beta-\beta^{\prime}$ (resinol) substructures (0) \\
\hline$C-\alpha$ & 4.85 & 71.9 & $\mathrm{C}_{\alpha}-\mathrm{H}_{\alpha}$ in $\beta-\mathrm{O}-4^{\prime}$ substructures $€$ \\
\hline $\mathrm{X}-2$ & 3.01 & 72.7 & $\mathrm{C}_{2}-\mathrm{H}_{2}$ in Xylopyranose \\
\hline $\mathrm{X}-3$ & 3.22 & 74.0 & $\mathrm{C}_{3}-\mathrm{H}_{3}$ in Xylopyranose \\
\hline $\mathrm{X}-4$ & 3.54 & 75.7 & $\mathrm{C}_{4}-\mathrm{H}_{4}$ in Xylopyranose \\
\hline$D-\beta^{\prime}$ & 4.11 & 79.4 & $\mathrm{C}_{\beta}^{\prime}-\mathrm{H}_{\beta}^{\prime}$ in $\beta-1^{\prime}$ (spriodienone) substructures (D) \\
\hline$\alpha-S A(\beta)$ & 4.92 & 79.5 & $\mathrm{C}_{\beta}-\mathrm{H}_{\beta}$ with a sulfonic acid group in $\alpha$ position \\
\hline$C-\beta\left(G^{\prime}\right)$ & 4.48 & 80.6 & $\mathrm{C}_{\beta}-\mathrm{H}_{\beta}$ in $\beta-\mathrm{O}-4^{\prime}$ substructures $€$ linked to a $\mathrm{G}^{\prime}$ unit \\
\hline$D-\alpha$ & 5.06 & 81.1 & $\mathrm{C}_{\alpha}-\mathrm{H}_{\alpha}$ in $\beta-1^{\prime}$ (spriodienone) substructures (D) \\
\hline$B E-\alpha$ & 4.74 & 81.4 & $\mathrm{C}_{\alpha}-\mathrm{H}_{\alpha}$ in benzyl ether LCC structures \\
\hline$C^{\prime}-\beta$ & 5.21 & 83.1 & $\begin{array}{l}\mathrm{C}_{\beta}-\mathrm{H}_{\beta} \text { in } \beta-\mathrm{O}-4^{\prime} \text { substructures } € \text { with oxidation in } \alpha \text { - } \\
\text { position }\end{array}$ \\
\hline$C-\beta$ (G, erytho) & 4.3 & 83.5 & $\begin{array}{l}C_{\beta}-H_{\beta} \text { in } \beta-O-4^{\prime} \text { substructures } € \text { linked to a } G \text { unit } \\
\text { erythron }\end{array}$ \\
\hline$C-\beta(G$, threo) & 4.3 & 83.1 & $\begin{array}{l}\mathrm{C}_{\beta}-\mathrm{H}_{\beta} \text { in } \beta-\mathrm{O}-4^{\prime} \text { substructures } € \text { linked to a } \mathrm{G} \text { unit } \\
\text { threo }\end{array}$ \\
\hline$A-\alpha$ & 4.63 & 85.1 & $C_{\alpha}-H_{\alpha}$ in $\beta-\beta^{\prime}$ (resinol) subsrtructures (A) \\
\hline
\end{tabular}


Table 3 continued.

\begin{tabular}{|c|c|c|c|}
\hline Crosspeak Label & H (ppm) & C (ppm) & Assignment \\
\hline$C-\beta(S)$ & 4.11 & 86.0 & $\mathrm{C}_{\beta}-\mathrm{H}_{\beta}$ in $\beta-\mathrm{O}-4^{\prime}$ substructures $(\mathrm{C})$ linked to a $\mathrm{S}$ unit \\
\hline$D-\alpha^{\prime}$ & 4.33 & 86.6 & 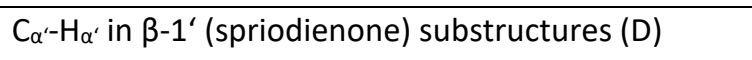 \\
\hline $\mathrm{E}-\alpha$ & 5.44 & 86.9 & $\mathrm{C}_{\alpha}-\mathrm{H}_{\alpha}$ in $\beta-5^{\prime}$ (phenylcoumaran) substructures (E) \\
\hline $\mathrm{X}-1$ & 4.27 & 101.7 & $\mathrm{C}_{1}-\mathrm{H}_{1}$ in Xylopyranose \\
\hline $\begin{array}{l}(1->4)-\beta-D- \\
\text { Manp }\end{array}$ & 4.66 & 101.6 & $\mathrm{C}_{1}-\mathrm{H}_{1}$ in $\beta$-D-Mannose with a 1-4-glycosidic linkage. \\
\hline$S-2,6$ & 6.64 & 103.6 & $\mathrm{C}_{2}-\mathrm{H}_{2}$ and $\mathrm{C}_{6}-\mathrm{H}_{6}$ in syringyl units (S) \\
\hline$S^{\prime}-2,6$ & 7.27 & 106.3 & $\mathrm{C}_{2}-\mathrm{H}_{2}$ and $\mathrm{C}_{6}-\mathrm{H}_{6}$ in oxidized syringyl units $(\mathrm{S})$ \\
\hline G-2 & 6.9 & 111.2 & $\mathrm{C}_{2}-\mathrm{H}_{2}$ in guaiacyl units $(\mathrm{G})$ \\
\hline $\mathrm{G}^{\prime}-2$ & 7.45 & 111.5 & $\mathrm{C}_{2}-\mathrm{H}_{2}$ in oxidized guaiacyl units $(\mathrm{G})$ \\
\hline$D-2^{\prime}$ & 6.25 & 113.5 & 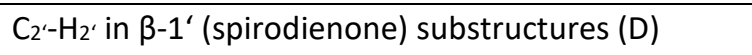 \\
\hline G-5 & 6.69 & 114.8 & $\mathrm{C}_{5}-\mathrm{H}_{5}$ in guaiacyl units $(\mathrm{G})$ \\
\hline G'-5 & 6.92 & 114.9 & $\mathrm{C}_{5}-\mathrm{H}_{5}$ in oxidized guaiacyl units $(\mathrm{G})$ \\
\hline D-6 & 6.09 & 118.8 & 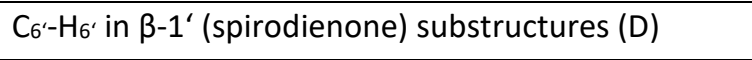 \\
\hline G-6 & 6.75 & 120.0 & $\mathrm{C}_{6}-\mathrm{H}_{6}$ in guaiacyl units (G) \\
\hline $\mathrm{G}^{\prime}-6$ & 7.55 & 123.2 & $\mathrm{C}_{6}-\mathrm{H}_{6}$ in oxidized guaiacyl units (G) \\
\hline Stilbene $(\alpha+\beta)$ & 6.99 & 126.3 & - \\
\hline $\mathrm{F}-\beta$ & 6.78 & 126.3 & $\mathrm{C}_{\beta}-\mathrm{H}_{\beta}$ in $\mathrm{p}$-hydroxycinnamyl aldehyde end groups $(\mathrm{F})$ \\
\hline $\mathrm{H}-2,6$ & 7.18 & 127.8 & $\mathrm{C}_{2}-\mathrm{H}_{2}$ and $\mathrm{C}_{6}-\mathrm{H}_{6}$ in $p$-hydroxyphenyl units $(\mathrm{H})$ \\
\hline$B-\beta$ & 6.45 & 128.4 & $\mathrm{C}_{\beta}-\mathrm{H}_{\beta}$ in $p$-hydroxycinnamyl alcohol end groups \\
\hline$B-\alpha$ & 6.33 & 130.0 & $\mathrm{C}_{\alpha}-\mathrm{H}_{\alpha}$ in $p$-hydroxycinnamyl alcohol end groups \\
\hline
\end{tabular}

\subsection{Volume integrals of characteristic lignin bonds.}

Table 4: HSQC volume integral intensity of characteristic structural motifs of all investigated lignin samples before and after EBI at $200 \mathrm{kGy}$. Intensities are normalized to lignin mass and representative C9 unit signals.

\begin{tabular}{|c|c|c|c|c|c|c|c|c|c|c|}
\hline Crosspeak Label & $\begin{array}{l}\text { MWL } \\
\text { A ref } \\
\text { (\%) }\end{array}$ & $\begin{array}{l}\text { MWL } \\
\text { A } 200 \\
(\%)\end{array}$ & $\begin{array}{l}\text { MWL } \\
\text { B ref } \\
\text { (\%) }\end{array}$ & $\begin{array}{l}\text { MWL } \\
\text { B 200 } \\
\text { (\%) }\end{array}$ & $\begin{array}{l}\text { MWL } \\
\text { C ref } \\
(\%)\end{array}$ & $\begin{array}{l}\text { MWL C } \\
200(\%)\end{array}$ & $\begin{array}{l}\text { KL ref } \\
\text { (\%) }\end{array}$ & $\begin{array}{l}\mathrm{KL} \\
200 \\
(\%)\end{array}$ & $\begin{array}{l}\text { SL ref } \\
\text { (\%) }\end{array}$ & SL 200 (\%) \\
\hline$A-\beta$ & 11.3 & 11.5 & 15.6 & 15.9 & 14.8 & 14.5 & 4.9 & 5.0 & 6.9 & 6.8 \\
\hline $\mathrm{MeO}$ & 559.3 & 559.7 & 601.9 & 583.8 & 598.6 & 602.2 & 462.2 & 453.9 & 762.7 & 759.4 \\
\hline B- - & - & - & - & - & - & - & 1.0 & 0.8 & - & - \\
\hline$C-\gamma$ & 63.3 & 64.3 & 62.1 & 61.9 & 67.2 & 61.6 & - & - & - & - \\
\hline$D-\beta$ & 0.6 & 0.8 & - & - & - & - & - & - & - & - \\
\hline$x-5$ & - & - & 35.7 & 34.8 & - & - & - & - & - & - \\
\hline$\alpha-S A(\alpha)$ & - & - & - & - & - & - & - & - & 0.8 & 0.9 \\
\hline A- -1 & 10.5 & 10.5 & 14.4 & 13.9 & 11.4 & 12.6 & 5.5 & 5.6 & 10.3 & 10.2 \\
\hline$A-\gamma 2$ & 12.2 & 11.1 & 17.7 & 16.0 & 14.5 & 13.9 & 6.4 & 6.6 & 8.0 & 7.5 \\
\hline$C-\alpha$ & 63.8 & 64.5 & 37.5 & 36.5 & 53.7 & 54.2 & 3.6 & 3.6 & 3.2 & 3.3 \\
\hline$x-2$ & 2.3 & 2.0 & 1.4 & 1.3 & 1.1 & 1.2 & 1.9 & 2.2 & - & - \\
\hline$x-3$ & 2.9 & 3.3 & 1.8 & 1.8 & 1.8 & 2.2 & 1.9 & 2.2 & - & - \\
\hline $\mathrm{X}-4$ & 7.3 & 7.7 & 3.2 & 2.8 & 4.1 & 3.9 & 2.3 & 2.7 & - & - \\
\hline
\end{tabular}


Table 4: continued.

\begin{tabular}{|c|c|c|c|c|c|c|c|c|c|c|}
\hline Crosspeak Label & $\begin{array}{r}\text { MWL } \\
\text { A ref } \\
(\%)\end{array}$ & $\begin{array}{r}\text { MWL } \\
\text { A } 200 \\
(\%)\end{array}$ & $\begin{array}{l}\text { MWL } \\
\text { B ref } \\
\text { (\%) }\end{array}$ & $\begin{array}{l}\text { MWL } \\
\text { B } 200 \\
\text { (\%) }\end{array}$ & $\begin{array}{l}\text { MWL } \\
\text { C ref } \\
(\%)\end{array}$ & $\begin{array}{l}\text { MWL C } \\
200(\%)\end{array}$ & $\begin{array}{l}\text { KL ref } \\
(\%)\end{array}$ & $\begin{array}{l}K L \\
200 \\
(\%) \\
\end{array}$ & $\begin{array}{l}\text { SL ref } \\
(\%)\end{array}$ & SL 200 (\%) \\
\hline$D-\beta^{\prime}$ & 0.6 & 0.7 & - & - & - & - & - & - & - & - \\
\hline$\alpha-S A(\beta)$ & - & - & - & - & - & - & - & - & 2.9 & 3.4 \\
\hline$C-\beta\left(G^{\prime}\right)$ & 2.5 & 3.0 & 16.2 & 15.5 & 4.3 & 4.6 & - & - & 2.2 & 2.2 \\
\hline$D-\alpha$ & 2.4 & 2.6 & 0.5 & 0.5 & 0.7 & 0.5 & - & - & - & - \\
\hline$B E-\alpha$ & - & - & - & - & - & - & 2.1 & 1.9 & 10.0 & 10.4 \\
\hline$C^{\prime}-\beta$ & 1.7 & 1.8 & 1.9 & 1.8 & 1.7 & 1.8 & - & - & 0.8 & 0.7 \\
\hline $\begin{array}{l}\mathrm{C}-\beta(\mathrm{G}, \\
\text { erytho+threo) }\end{array}$ & 15.7 & 15.4 & 14.2 & 14.3 & 11.6 & 10.9 & 0.6 & 0.7 & - & - \\
\hline$A-\alpha$ & 16.3 & 16.7 & 20.4 & 19.4 & 17.9 & 18.6 & 5.6 & 5.6 & 11.7 & 10.9 \\
\hline$C-\beta(S)$ & 34.9 & 35.9 & 32.6 & 31.9 & 31.9 & 32.1 & 0.7 & 0.6 & 0.6 & 0.6 \\
\hline$D-\alpha^{\prime}$ & 4.1 & 4.5 & 4.5 & 4.4 & 3.3 & 4.3 & 1.3 & 1.4 & 2.4 & 2.3 \\
\hline$E-\alpha$ & 3.5 & 3.3 & 4.0 & 3.8 & 2.8 & 3.1 & 0.2 & 0.2 & 0.2 & 0.3 \\
\hline $\mathrm{X}-1$ & - & - & - & - & - & - & 1.1 & 1.3 & - & - \\
\hline $\begin{array}{l}(1->4)-\beta-D- \\
\text { Manp }\end{array}$ & - & - & 2.5 & 2.7 & - & - & - & - & - & - \\
\hline$S-2,6$ & 113.7 & 111.8 & 112.4 & 109.4 & 112.8 & 112.6 & 149.9 & 152.3 & 170.2 & 169.3 \\
\hline$S^{\prime}-2,6$ & 11.1 & 12.9 & 9.5 & 11.1 & 11.2 & 11.4 & 4.8 & 4.5 & 21.4 & 22.0 \\
\hline G-2 & 36.2 & 36.2 & 37.4 & 38.0 & 36.0 & 35.6 & 22.7 & 21.6 & - & - \\
\hline $\mathrm{G}^{\prime}-2$ & 1.4 & 1.5 & 1.6 & 1.7 & 2.0 & 2.4 & - & - & 4.2 & 4.3 \\
\hline D-2' & 1.2 & 1.3 & - & - & - & - & - & - & - & - \\
\hline G-5 & 21.7 & 22.8 & 29.9 & 28.5 & 33.0 & 36.0 & 28.1 & 29.0 & 87.5 & 87.6 \\
\hline $\mathrm{G}^{\prime}-5$ & 14.9 & 16.5 & 15.6 & 15.5 & 15.6 & 17.3 & - & - & - & - \\
\hline D-6' & 1.0 & 1.2 & - & - & - & - & - & - & - & - \\
\hline G-6 & 29.1 & 32.6 & 36.7 & 36.6 & 29.2 & 37.3 & 28.5 & 27.8 & 19.9 & 20.1 \\
\hline$G^{\prime}-6$ & 0.9 & 1.0 & 0.9 & 0.9 & 2.4 & 2.5 & 0.9 & 1.0 & 1.7 & 1.7 \\
\hline Stilbene $(\alpha+\beta)$ & - & - & - & - & - & - & 3.9 & 4.1 & - & - \\
\hline$F-\beta$ & 1.3 & 3.2 & 2.2 & 3.1 & 1.3 & 4.4 & - & - & - & - \\
\hline $\mathrm{H}-2,6$ & 2.5 & 3 & - & - & 3.6 & 8.8 & - & - & 8.9 & 8.9 \\
\hline$B-\beta$ & 4.6 & 4.6 & - & - & - & - & - & - & - & - \\
\hline$B-\alpha$ & 4 & 2 & - & - & - & - & - & - & - & - \\
\hline
\end{tabular}

\subsection{HSQC-NMR spectra}

Figures 1-10 show the HSQC-NMR spectra of all tested lignin samples. Reference and irradiated lignin variant are compared based on an overview and zoom cuts for linkage and aromatic region, respectively. 


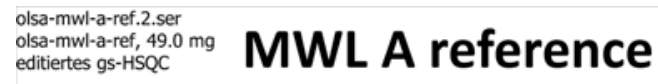

\section{F. sylvatica}

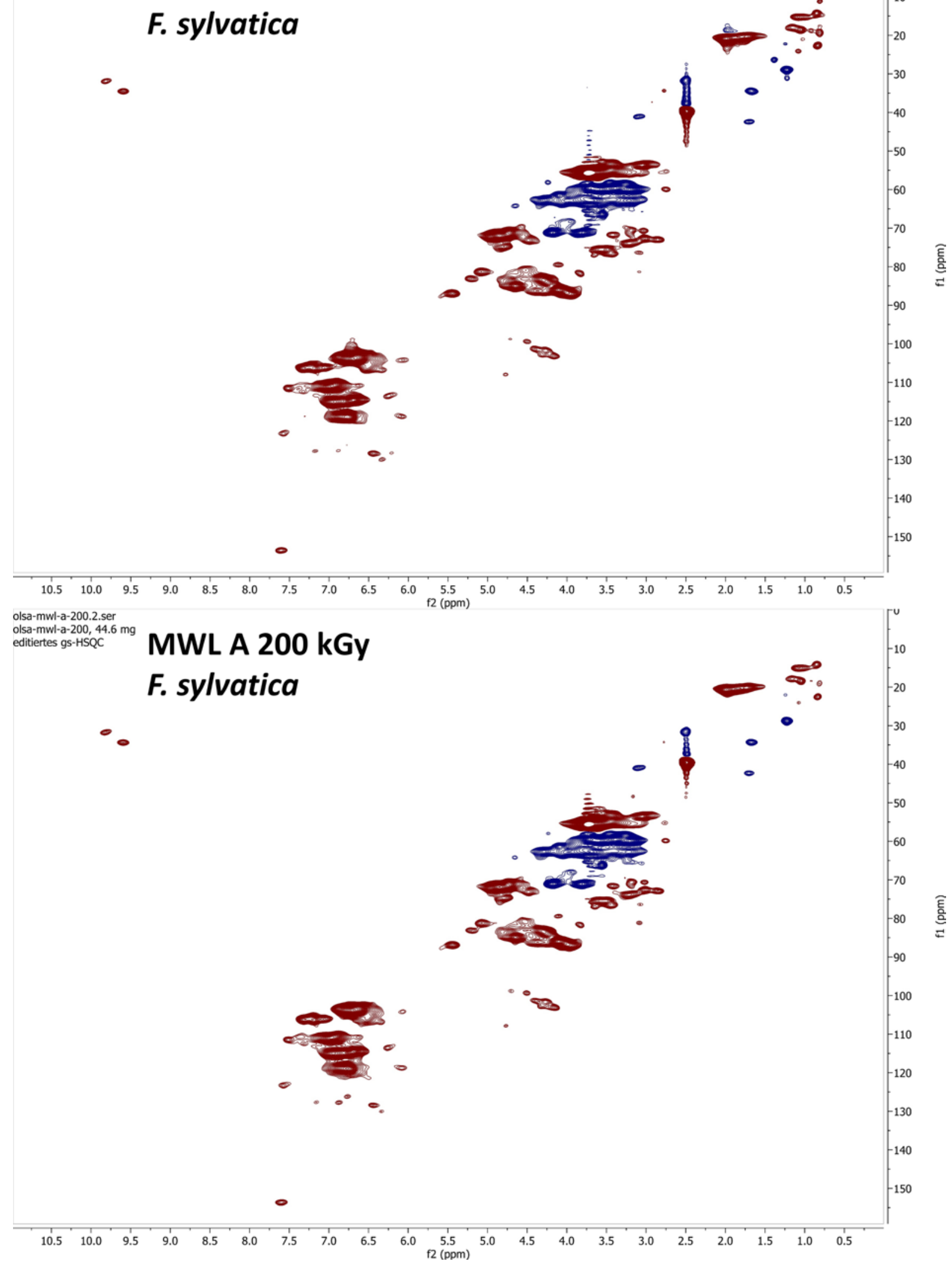

Figure 1: MWL A overview. 


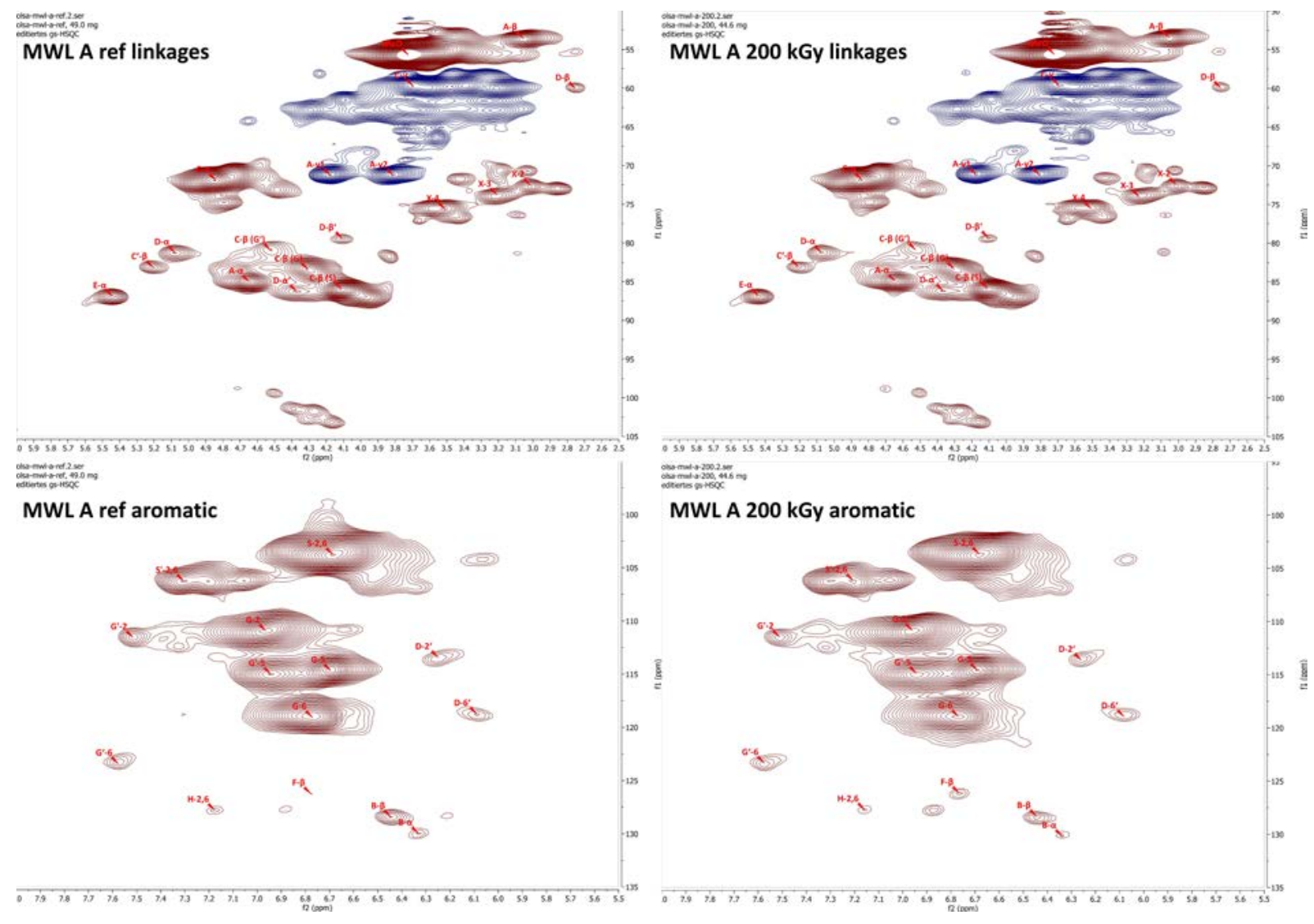

Figure 2: MWL A zoomed regions. 


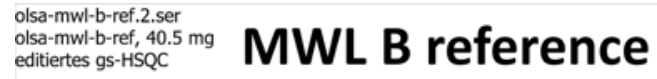

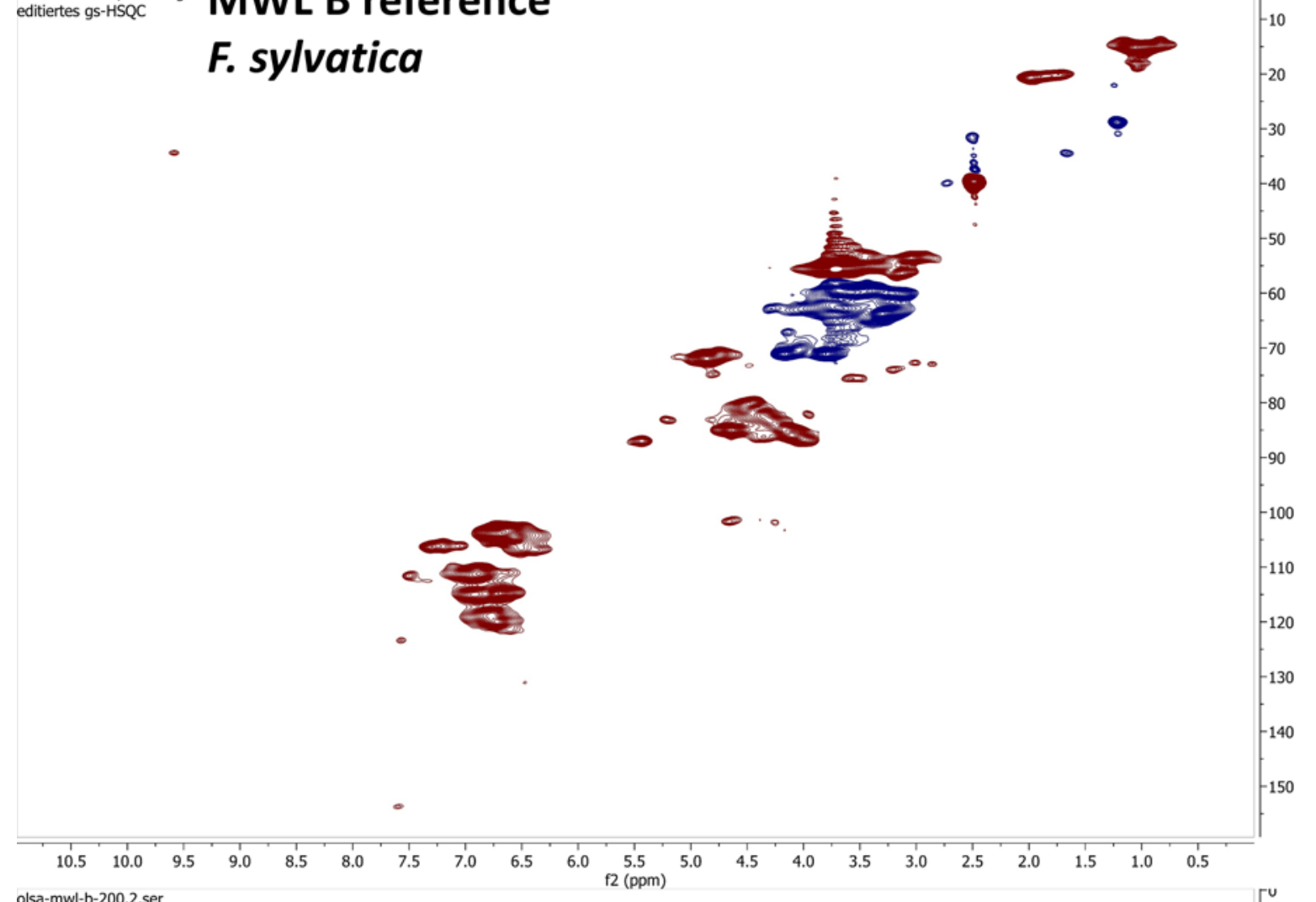

olsa-mwl-b-200.2.ser

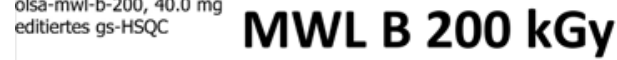

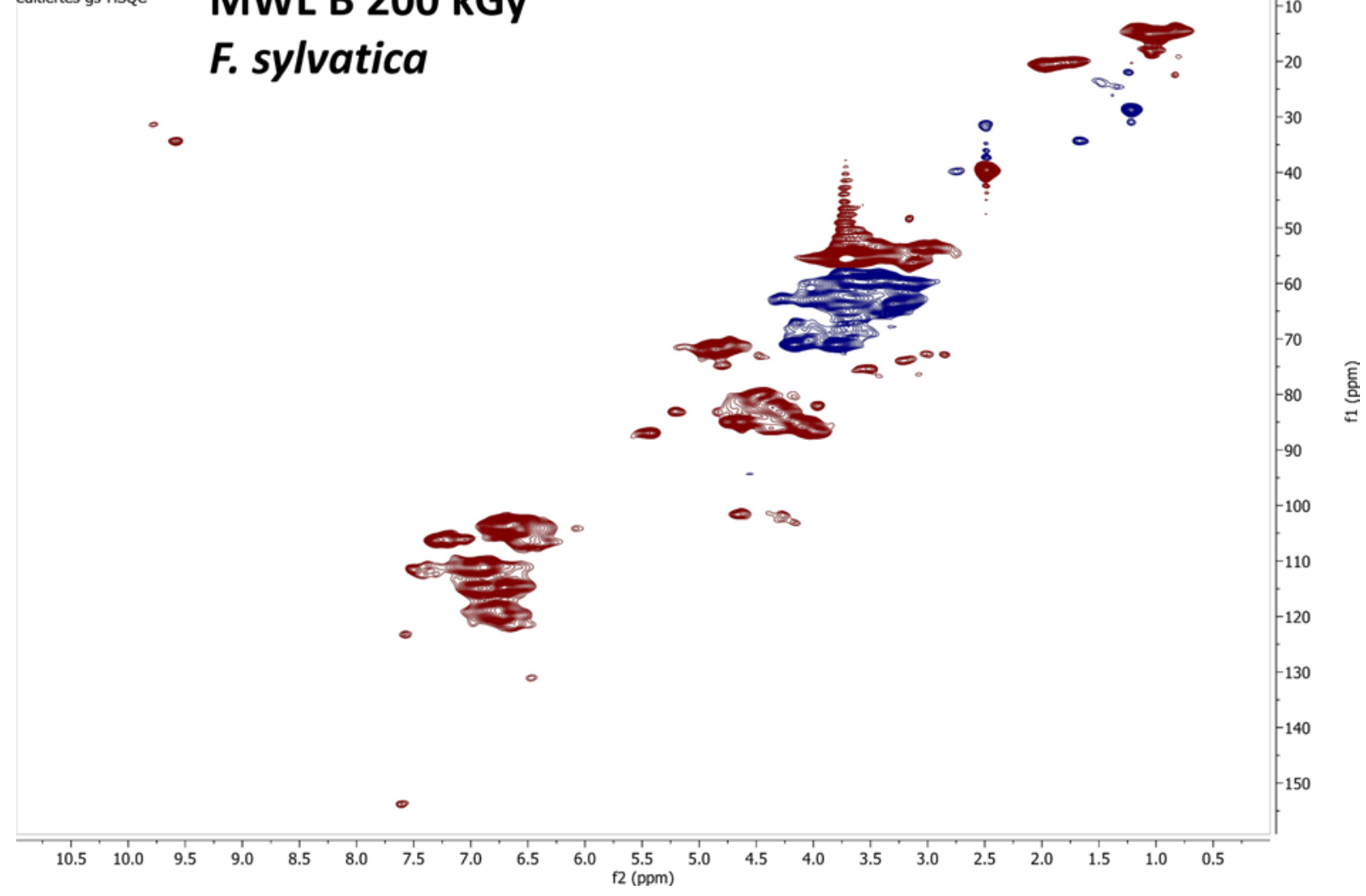

Figure 3 MWL B overview. 


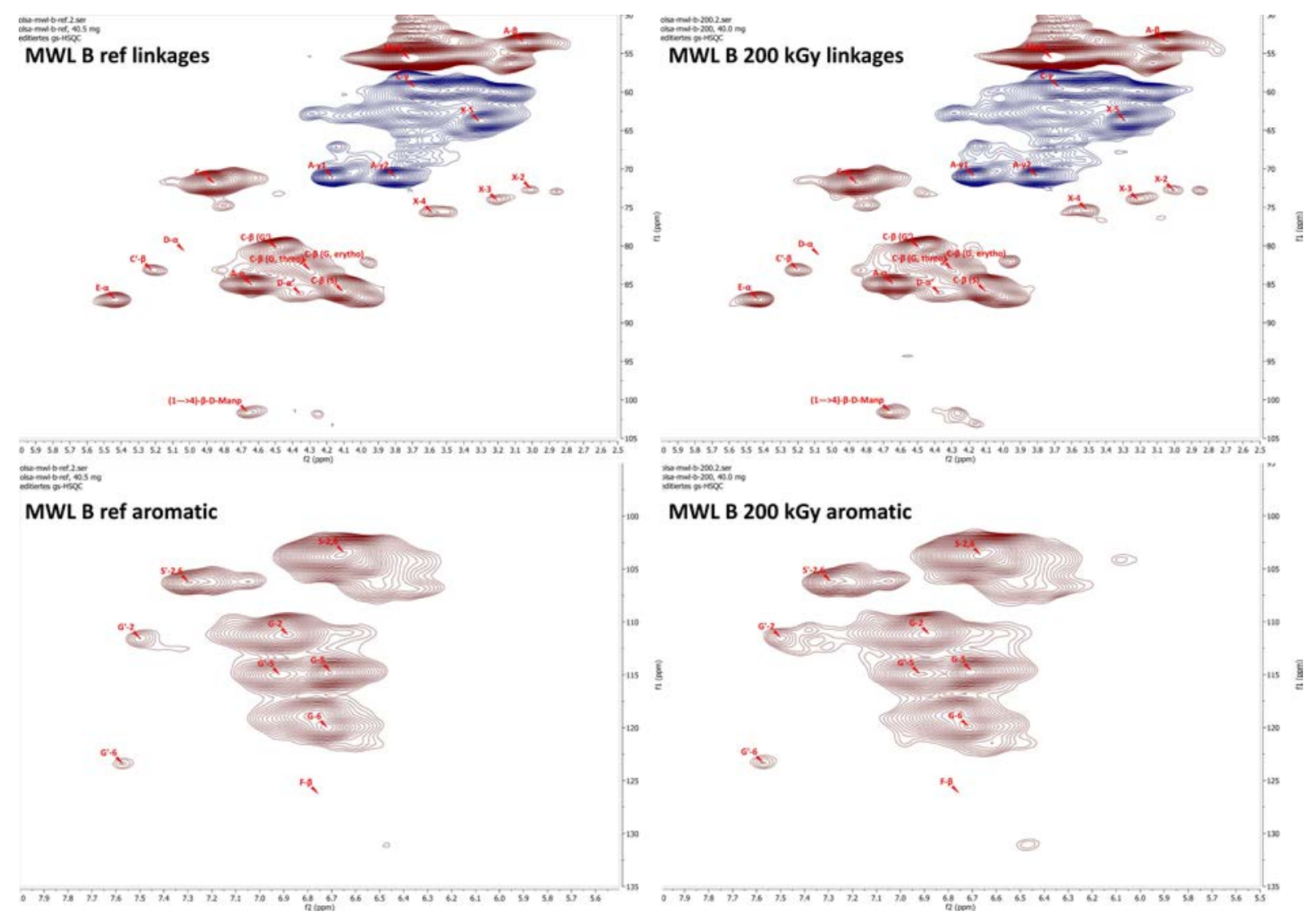

Figure 4: MWL B zoomed regions. 


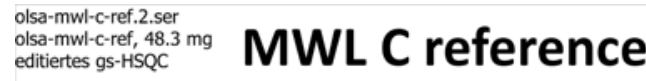

\section{E. globulus}

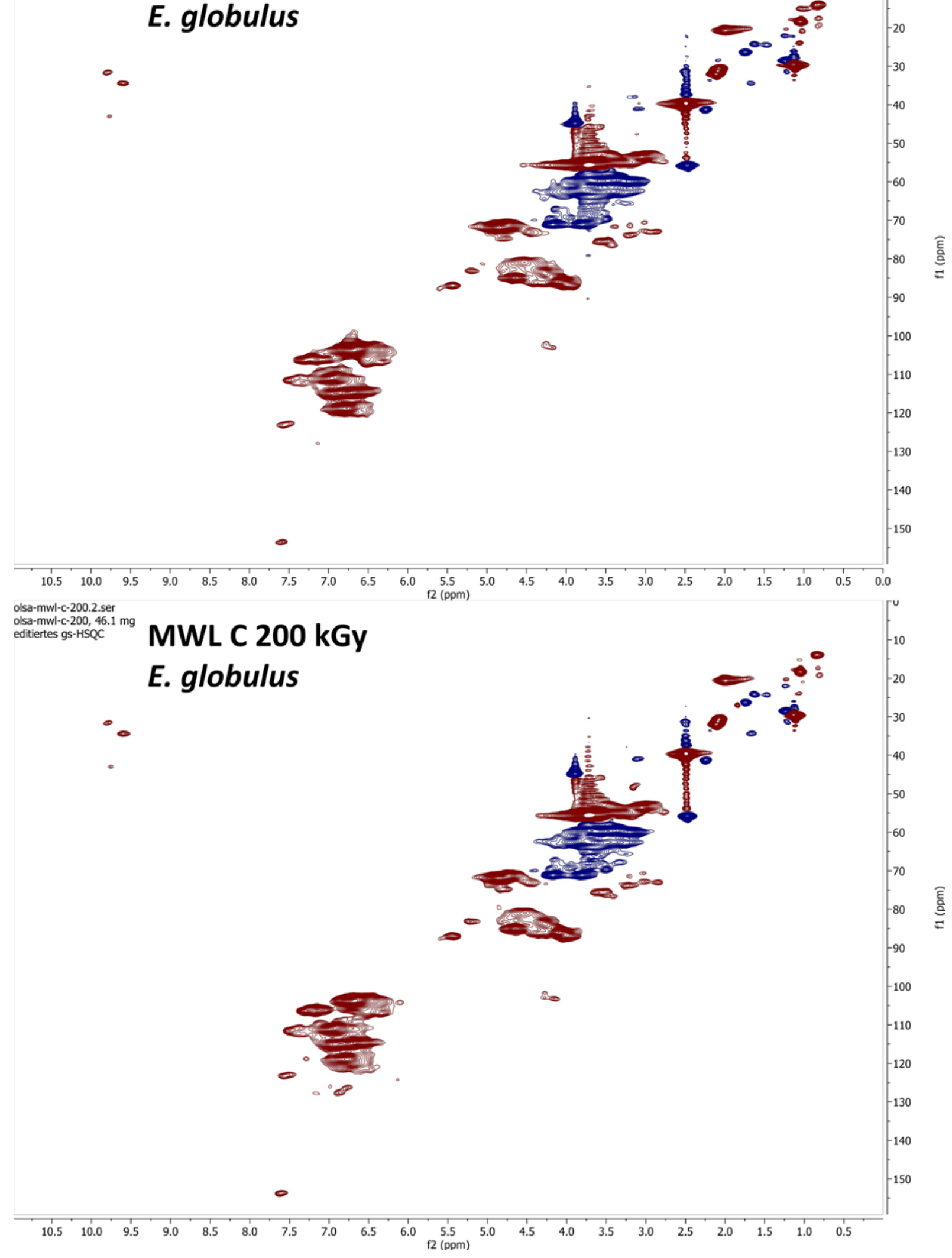

Figure 5: MWL C overview. 


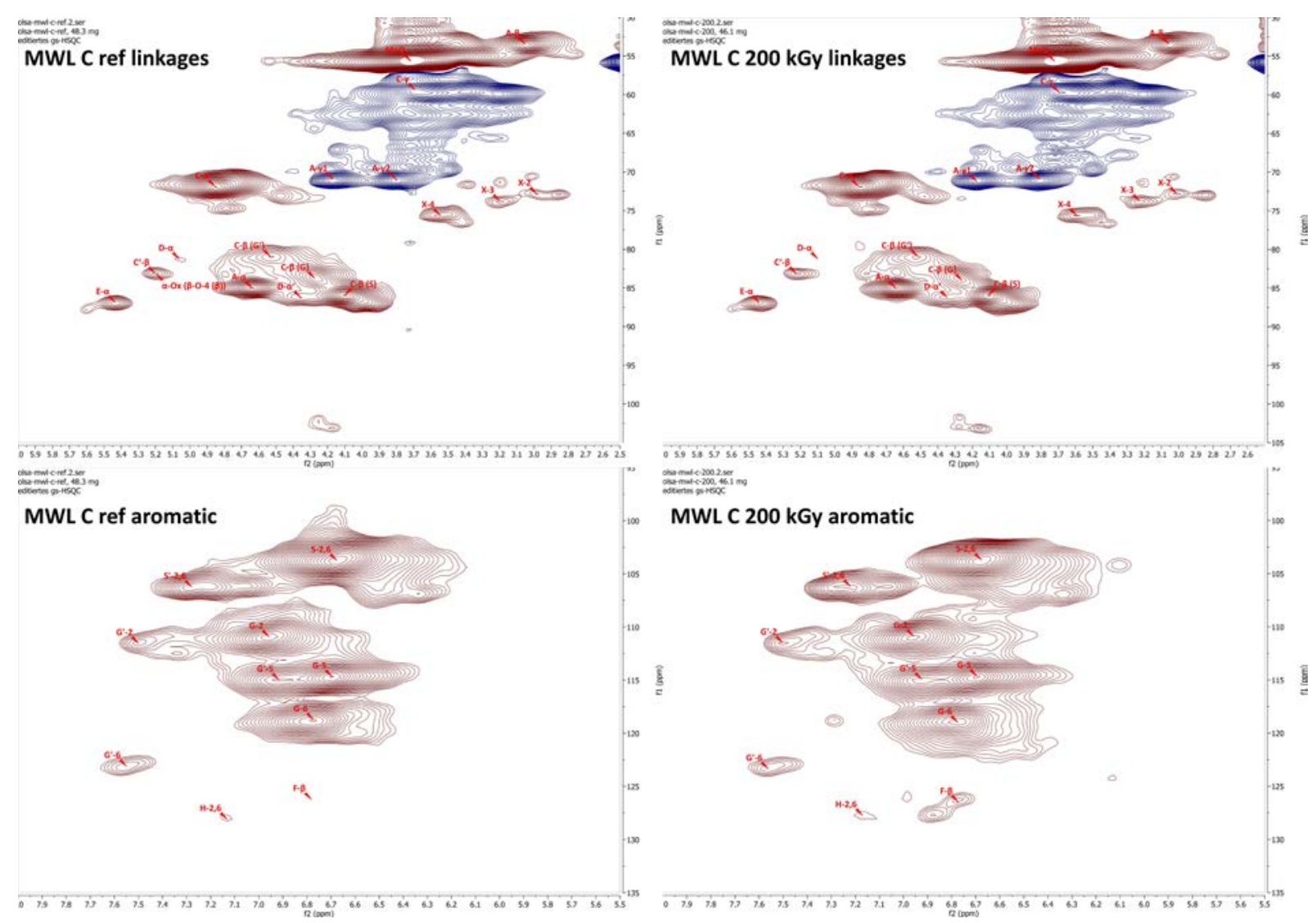

Figure 6: MWL C zoomed regions. 
E. globulus

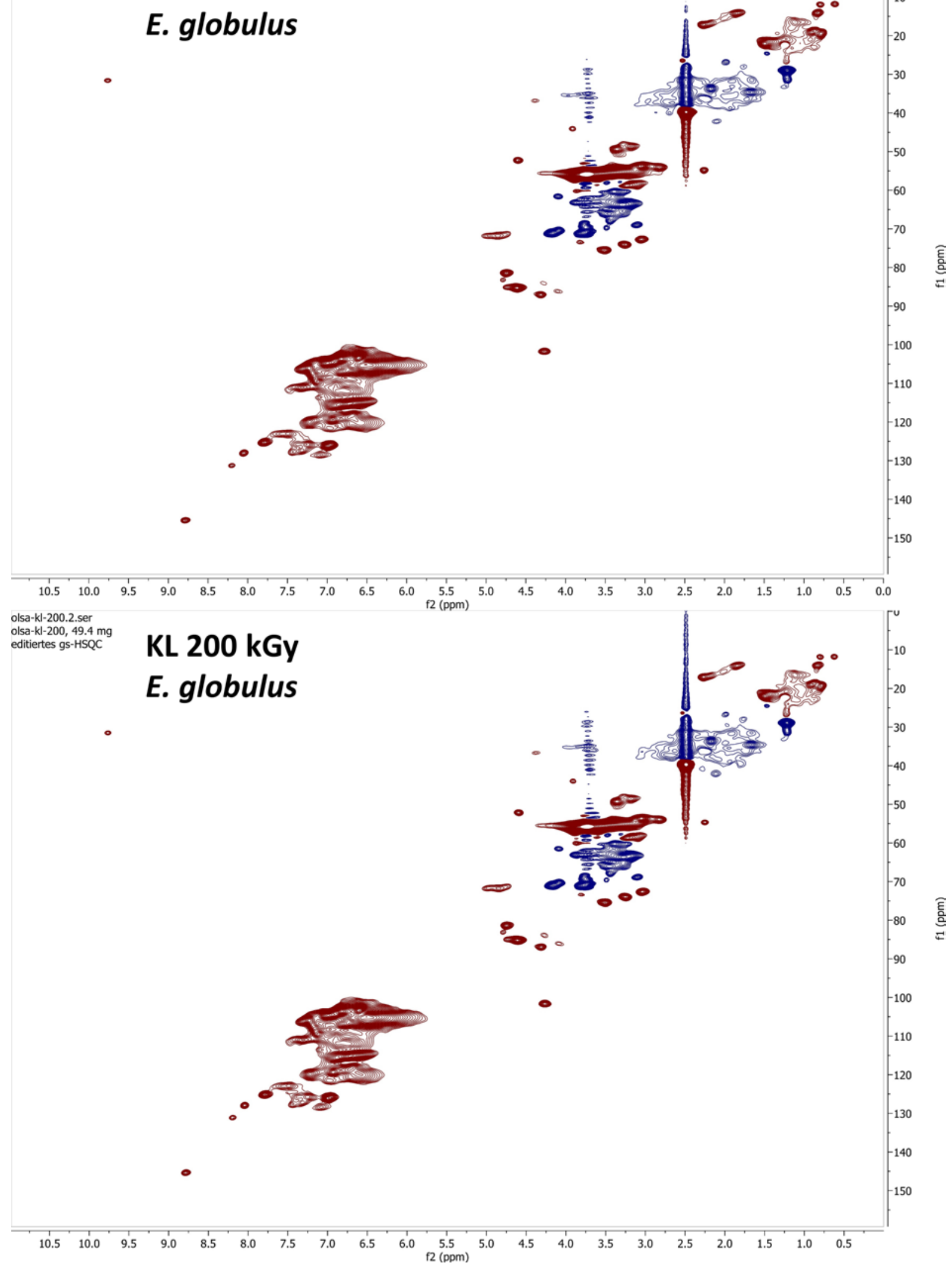

Figure 7: KL overview. 


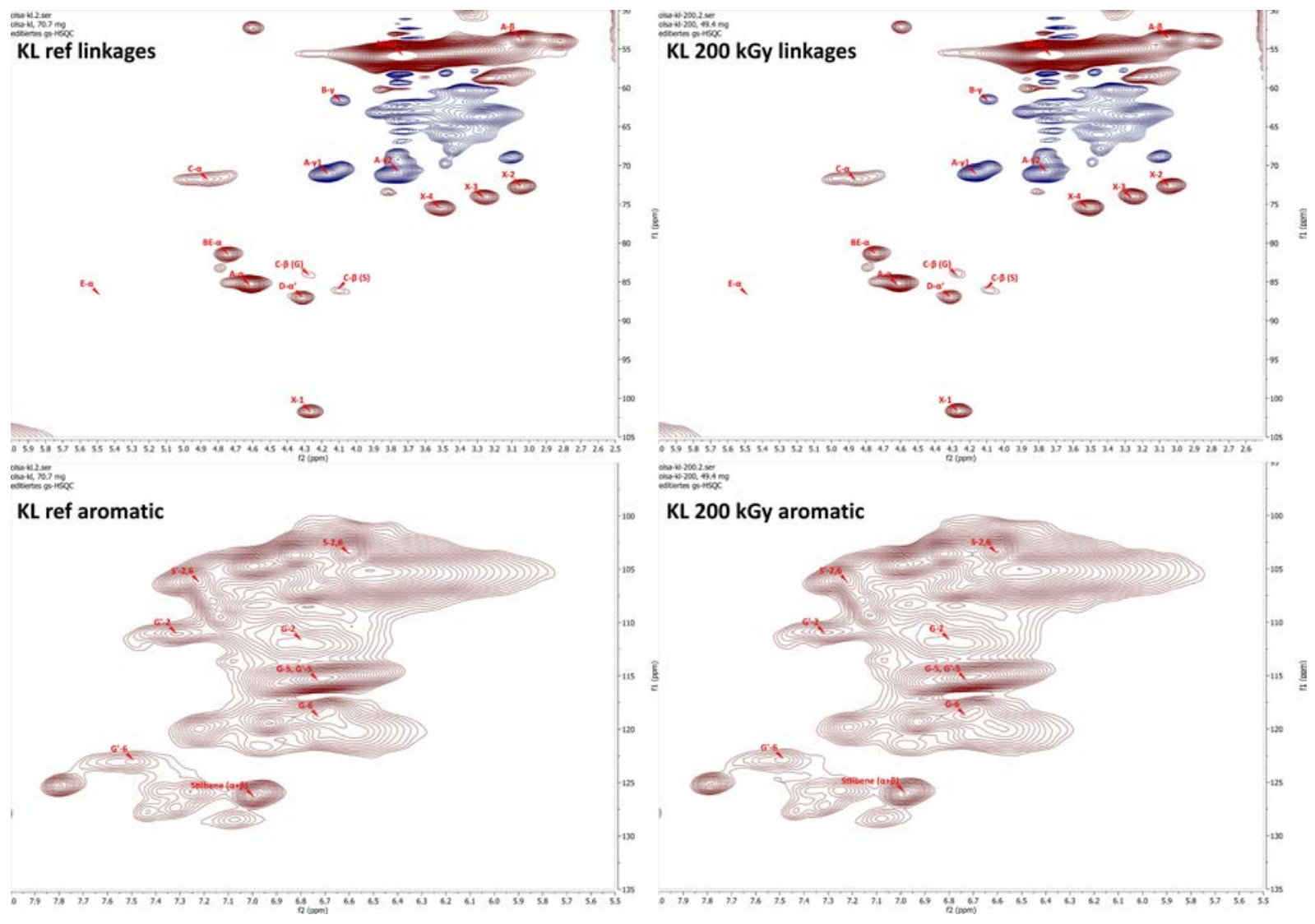

Figure 8: KL zoomed sections. 


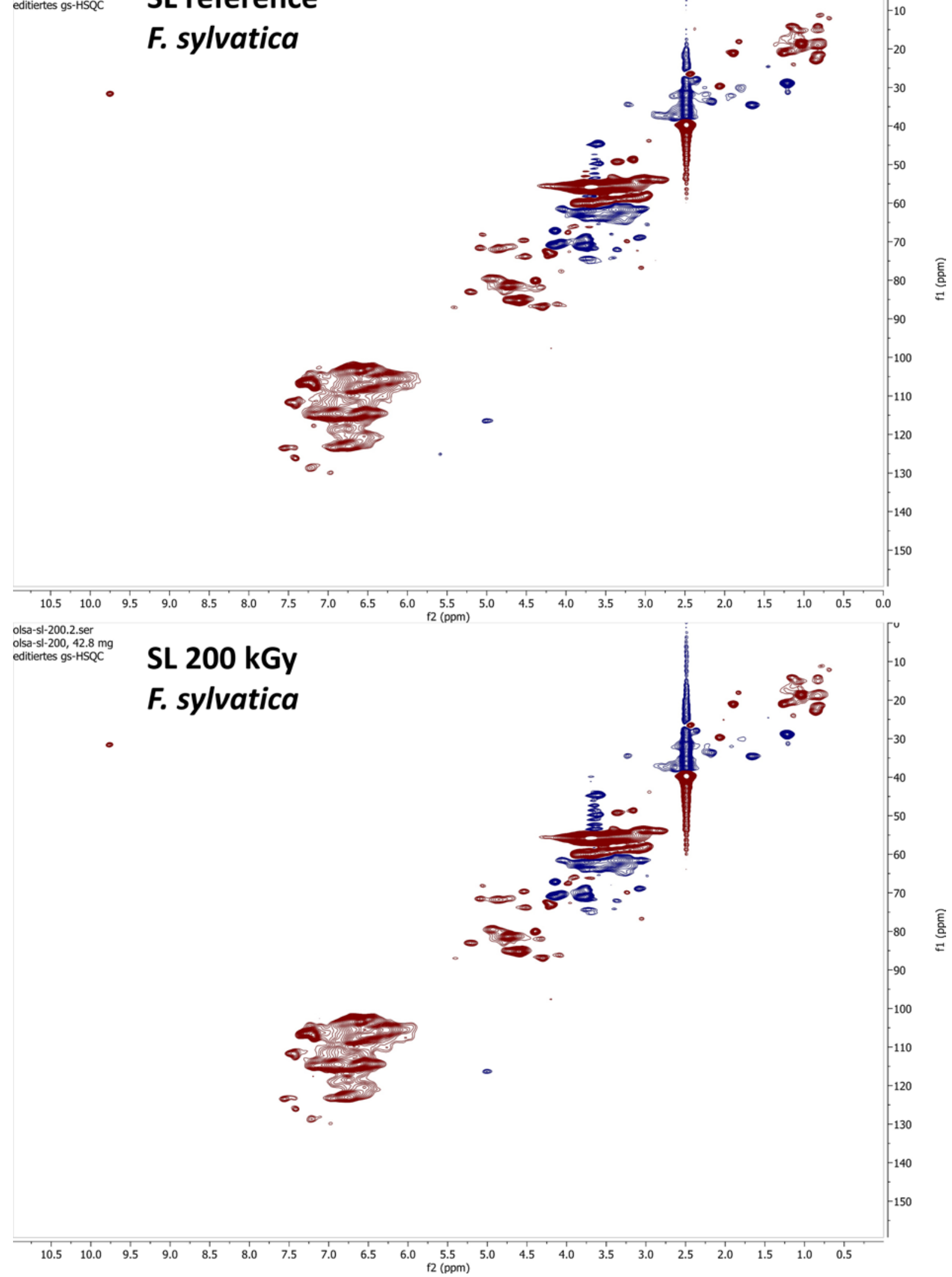

Figure 9: SL overview. 


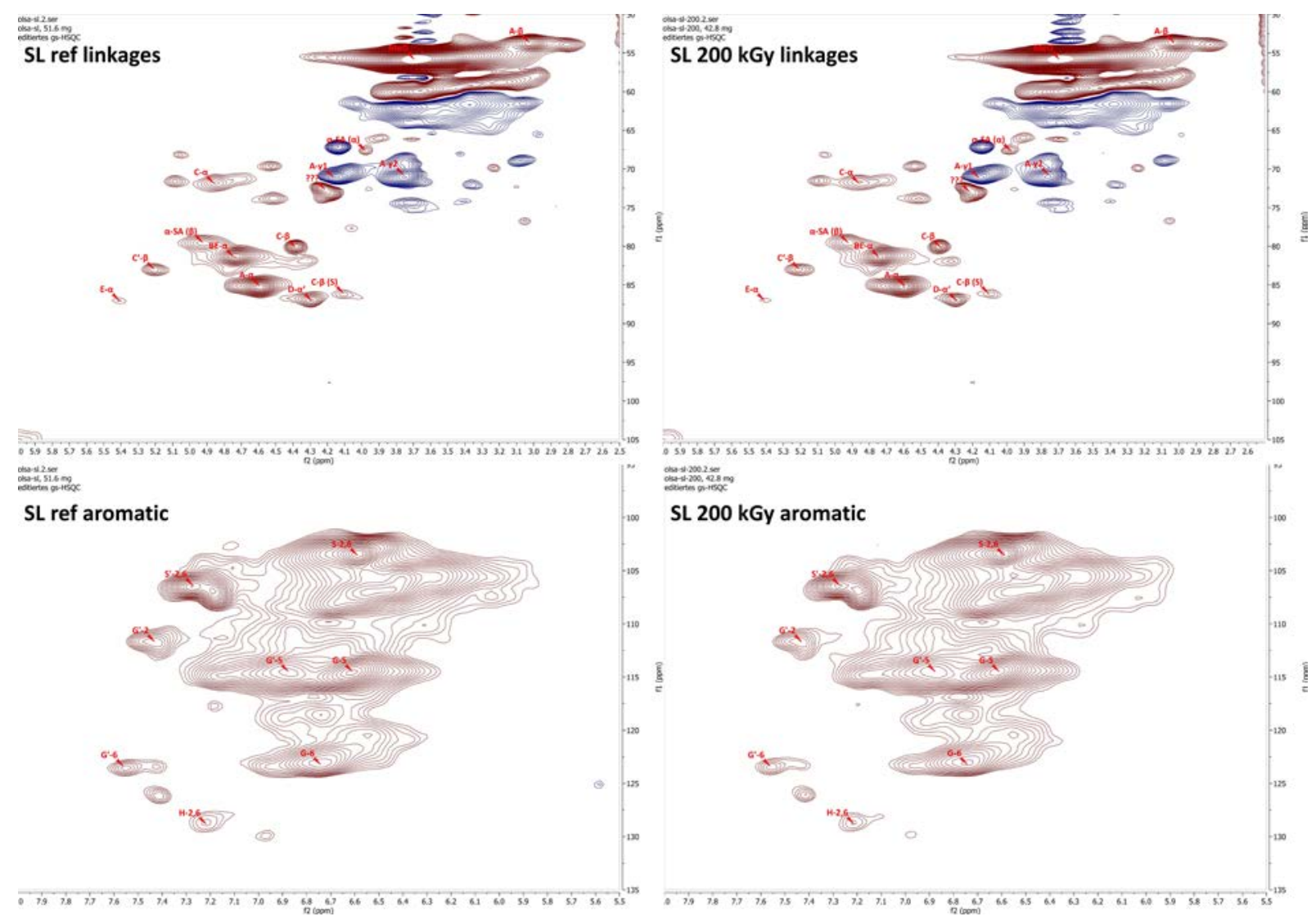

Figure 10: SL zoomed regions. 